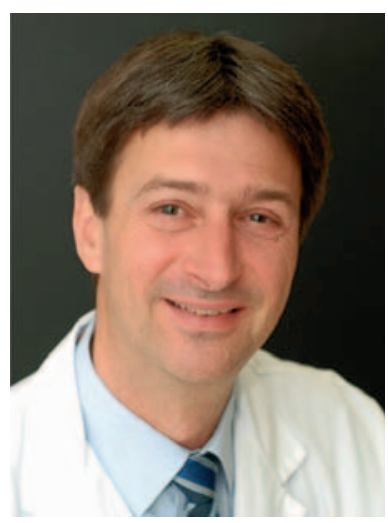

Prof. Dr. med. Christoph M. Schempp, Freiburg i.Br., Deutschland

\section{Komplementärmedizin in der Dermatologie: Nicht Alternative, sondern Ergänzung der akademischen Medizin}

Komplementärmedizinische Therapieansätze werden in der Dermatologie, anders als in anderen Fachrichtungen wie der Pädiatrie oder Urologie, eher selten eingesetzt. Bei genauerer Betrachtung hat aber gerade die Phytotherapie schon längst Einzug in die dermatologische Therapie gehalten. So wurden viele in der äusserlichen Anwendung etablierte Stoffe ursprünglich aus Pflanzen gewonnen - wie z.B. die Salicylsäure (zuerst aus Weidenrinde isoliert, verwendet als Keratolytikum), das Cignolin (ein aus der Rinde des Tropenbaumes Andira araroba isoliertes Anthralin, verwendet zur Behandlung der Schuppenflechte) oder das 8-Methoxypsoralen (ein Furocumarin aus der Ammipflanze, das für die PUVA-Therapie - d.h. Psoralen plus Ultraviolettlicht des langwelligen Bereichs A - verwendet wird). Natürliches Menthol bindet an den TRPM8-Rezeptor und wirkt so kühlend und juckreizlindernd.

In den letzten Jahren gab es aber auch Neuzulassungen von dermatologischen Arzneimitteln auf Naturstoffbasis. So wurde ein Grüntee-Extrakt $\left(\right.$ Veregen ${ }^{\circledR}$ ) zur Behandlung von Kondylomen zugelassen. Der isolierte Reinstoff Ingenolmebutat aus der Garten-Wolfsmilch (Picato ${ }^{\circledR}$ ) wird zur Behandlung von aktinischen Keratosen eingesetzt, und ein mit einem Trockenextrakt aus der Birkenrinde hergestelltes Oleogel (Episalvan ${ }^{\circledR}$ ) wurde 2016 von der Europäischen
Arzneimittel-Agentur (EMA) für die Behandlung von Verbrennungen und oberflächlichen Wunden zugelassen.

Die molekulare Medizin hat in den letzten Jahren nicht nur zu einem vertieften Verständnis der Pathogenese vieler Hauterkrankungen geführt, sondern auch zur Entwicklung sehr spezifischer, hochwirksamer systemischer Therapien. Individualisierte, massgeschneiderte molekulare Therapien etablieren sich zunehmend auch in der Dermatologie. Dies hat vor allem bei der Behandlung von Hautkrebs zu einer Revolutionierung der Therapiemöglichkeiten geführt. So wurde der Hedgehog-Signalweg-Inhibitor Vismodegib als erster Wirkstoff für die innerliche Behandlung des fortgeschrittenen Basalzellkarzinoms zugelassen. Neue Immuntherapien wie BRAF-Inhibitoren, MEK-Inhibitoren und CD1-Inhibitoren haben die Therapie des fortgeschrittenen malignen Melanoms so verbessert, dass das metastasierte Melanom heute kein unausweichliches Todesurteil mehr darstellt. In ähnlicher Weise stehen für die Behandlung der Psoriasis seit einigen Jahren mehrere antiinflammatorische Zytokin-Inhibitoren mit günstigem Nutzen-Risiko-Profil zur Verfügung.

Braucht es dann auf dem Hintergrund dieser Fortschritte der molekularen Medizin in der Dermatologie überhaupt noch komplementärmedizinische Therapien? Die Antwort lautet eindeutig: ja! Komplementärmedizinische Therapien, vor allem die Phytotherapie, lassen sich sogar sehr elegant mit massgeschneiderten molekularen Therapien kombinieren. Gerade der Trend zu einer immer mehr individualisierten Medizin verlangt danach, dem Bedürfnis des Patienten nach ergänzenden sanfteren, «grünen» Therapien entgegenzukommen. Umfragen zeigen immer wieder, dass ein hoher Teil der Patienten und der Bevölkerung sich komplementärmedizinische Therapien wünscht - meistens in der Hoffnung, damit Nebenwirkungen stark wirksamer Medikamente zu verringern bzw. auch die Dosis und Frequenz schulmedizinischer Therapien reduzieren zu können. Und genau dort liegt die Stärke der komplementärmedizinischen Verfahren. Aus der Vielfalt dieser Therapieansätze werden im Rahmen des Themenschwerpunktes «Dermatologie» in dieser Ausgabe der Schweizerischen Zeitschrift für GANZHEITSMEDIZIN die wichtigsten vorgestellt.

Die Biologin Dr. Wölfle beschreibt die Flavonoide als besonders interessante sekundäre Pflanzenstoffe für die Haut [1]. Insbesondere das Luteolin wurde in den letzten Jahren intensiv dermatologisch erforscht. Es hat ein grosses Potenzial in der Vorbeugung, aber auch in der Behandlung exogen-toxischer Einflüsse. Hier sind insbesondere die UVStrahlung und das allergische Kontaktekzem zu nennen.

\section{KARGER}

(C) 2017 S. Karger GmbH, Freiburg
Klinik für Dermatologie und Venerologie, Universitätsklinikum Freiburg

Hauptstrasse 7, 79104 Freiburg, Deutschland

christoph.schempp@uniklinik-freiburg.de 
Der Apotheker Dr. Kohlhase berichtet über die kürzlich entdeckten Bitterstoff-Rezeptoren der Haut [2]. Die Haut kann tatsächlich «schmecken», und innerlich sowie äusserlich angewendete Bitterstoffe vermitteln heilsame Wirkungen an der Haut.

In dem Beitrag von Prof. Gündling wird ein Überblick über die bewährten, traditionellen phytotherapeutischen Behandlungsverfahren in Form kurzer Pflanzenporträts gegeben [3]. Auch die innerliche Behandlung mit pflanzlichen Zubereitungen bzw. Kräutertees wird angesprochen.

Dr. Jachens, Hautarzt und Leiter des anthroposophischdermatologischen Arbeitskreises der Gesellschaft Anthroposophischer Ärzte in Deutschland (GAÄD), stellt das Konzept der Anthroposophischen Medizin in der Dermatologie vor [4]. Dieser auch didaktisch interessante Ansatz eröffnet eine integrative Sicht auf das Hautorgan.

Naturgemäss kann ein solcher Themenschwerpunkt nicht alle Aspekte dieses wichtigen Themas abdecken. Wir hoffen, Ihnen dennoch einen informativen, lehrreichen und auch für Ihre Praxis hilfreichen Überblick zusammengestellt zu haben. Ich persönlich freue mich, dass unsere eidgenössischen Nachbarn die Komplementärmedizin so vorbildlich in die medizinische Versorgung integriert haben und hoffe, dass auch wir in Deutschland diesem Vorbild baldmöglichst folgen werden.

In diesem Sinne: Viel Spass beim Lesen!

\section{Literatur}

1 Wölfle U, Schempp CM: Flavonoide als vielseitige Wirkstoffe für die Haut - Fokus Luteolin. Schweiz Z Ganzheitsmed 2017;29:DOI: 10.1159/ 0004777788.

2 Kohlhase M, Wölfle U, Schempp CM: Bitterstoffe als belebende Wirkstoffe für die Haut. Schweiz Z Ganzheitsmed 2017;29:DOI: 10.1159/ 000477568.

3 Gündling PW: Einsatzmöglichkeiten der Phytotherapie bei Hautkrankheiten. Schweiz Z Ganzheitsmed 2017;29:DOI: 10.1159/000477478.

4 Jachens L: Der ganzheitliche Ansatz der Anthroposophischen Medizin in der Dermatologie. Schweiz Z Ganzheitsmed 2017;29:DOI: 10.1159/ 000477553. 